\title{
The SAX mission for wide-band X-ray astronomy $(*)\left({ }^{* *}\right)$
}

\author{
G. Di CocCO \\ for the SAX collaboration(***) \\ Istituto TESRE/CNR - via P. Gobetti 101, 40129 Bologna, Italy
}

(ricevuto il 4 Aprile 1996; approvato il 24 Maggio 1996)

\begin{abstract}
Summary. - The SAX (Satellite per Astronomia X) mission is a major joint program of the Italian Space Agency (ASI) and the Netherlands Agency for Space Programs (NIVR). In the framework of past and future X-ray missions the SAX satellite, launched in April 1996, stands out for its very wide spectral coverage from 0.1 to $200 \mathrm{keV}$, with well-balanced performances of the low-energy and high-energy instrumentation. The sensitivity of the scientific payload will allow the exploitation of the full band of SAX also for weak sources (1/20 of 3C273), opening new perspectives in the study of spectral shape and variability of several classes of objects. In this paper we describe the main aspects of the mission, the instruments, the scientific objectives and operations.
\end{abstract}

PACS 96.40 - Cosmic rays.

PACS 95.55 - Astronomical and space-research instrumentation.

PACS 01.30.Cc - Conference proceedings.

\section{1. - Outline of the mission}

The study of the spectral behaviour of celestial sources over a wide range of energies is of primary importance in understanding comprehensively the emission mechanisms that, in several instances, produce spectral features localized in different regions of the electromagnetic spectrum.

$\left({ }^{*}\right)$ Paper presented at the VII Cosmic Physics National Conference, Rimini, October 26-28, 1994.

$\left({ }^{* *}\right)$ The author of this paper has agreed to not receive the proofs for correction.

$(* * *)$ SAX Consortium:

Istituto per le Tecnologie e lo Studio Radiazioni Extraterrestri, CNR, Bologna (Istituto TESRE);

Istituto Astrofisica Spaziale, CNR, Frascati, (IAS);

Istituto Fisica Cosmica e Tecnologie Relative, CNR (IFCTR) ed Unità GIFCO, Milano;

Istituto di Fisica Cosmica ed Appl. Informatica (IFCAI) ed Unità GIFCO, Palermo;

Istituto Osservatorio Astronomico, Università di Roma "La Sapienza";

Space Research Institute of Utrecht/SRON (SRU), The Netherlands;

Space Science Departments (SSD), ESA, Noordwijk, The Netherlands. 


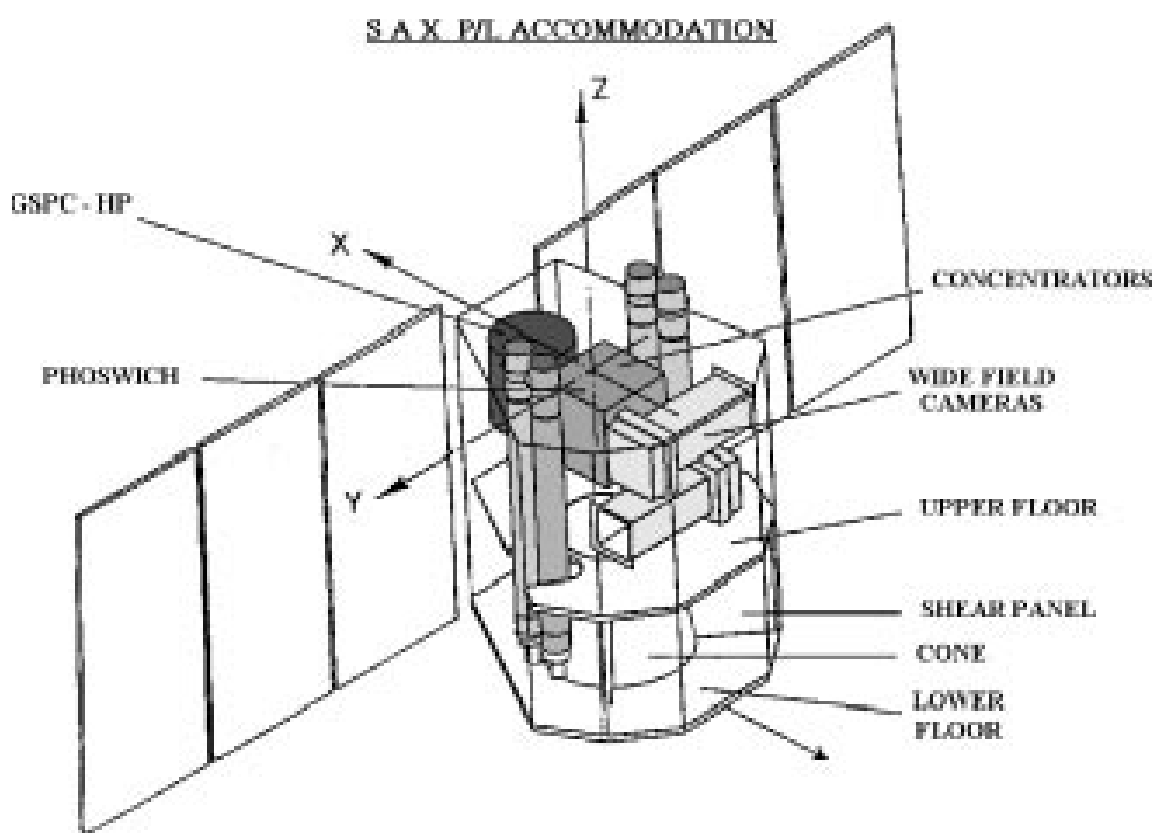

Fig. 1. - SAX instruments accommodation.

The Italian-Dutch X-ray Satellite SAX will be the first X-ray mission that will have the capability of observing sources over more than three decades of energy -from 0.1 to $200 \mathrm{keV}$ - with a relatively large area, a good energy resolution, associated with imaging capabilities (resolution of about $1^{\prime}$ ) in the range of $0.1-10 \mathrm{keV}$.

The instrument complement dedicated to such purpose (fig. 1) is composed of a medium-energy (1-10 keV) concentrator optics/spectrometer, MECS, consisting of three units, a low-energy (0.1-10 keV) concentrator optics/spectrometer, LECS, a highpressure gas scintillation proportional counter (3-120 keV), HPGSPC, and a Phoswich Detector System (15-300 keV), PDS, all of which have narrow fields and point in the same direction (Narrow-Field Instruments, NFI).

The other characterization of the mission is its capability of monitoring large regions of the sky with a resolution of $5^{\prime}$ in the range $2-30 \mathrm{keV}$ to study long-term variability of sources down to $1 \mathrm{mCrab}$ and to detect X-ray transient phenomena. This is realized by means of two coded mask proportional counters (Wide Field Cameras, WFC) pointing in diametrically opposed directions perpendicular to the NFI (fig. 1). Finally, the anticoincidence scintillator shields of the PDS will be used as a gamma-ray burst monitor in the range $60-600 \mathrm{keV}$. The main characteristics of the satellite onboard experiments are given in table I.

SAX has been launched by an ATLAS G-Centaur into a $600 \mathrm{~km}$ orbit at 3 degrees inclination in April 1996.

During each orbit up to 450 Mbits of data will be stored onboard and relayed to ground on the station contact. The average data rate available to instruments will be about $60 \mathrm{kbits} / \mathrm{s}$, but peak rates of up to $1100 \mathrm{kbits} / \mathrm{s}$ can be retained. The satellite passes above the ground station, placed near the equator in Malindi, once every orbit. This will allow a prompt operation and control on the satellite. 
TABLE I. - SAX instruments.

\begin{tabular}{|c|c|c|c|c|c|}
\hline Instrument & $\begin{array}{c}\text { Range } \\
\text { (keV) }\end{array}$ & $\begin{array}{c}\text { FOV } \\
\left({ }^{\circ} \mathrm{FWHM}\right)\end{array}$ & $\begin{array}{l}\text { Angular res. } \\
\text { (arcmin) }\end{array}$ & $\begin{array}{l}\text { Area } \\
\left(\mathrm{cm}^{2}\right)\end{array}$ & $\begin{array}{l}\text { Energy res. } \\
\text { (\% FWHM) }\end{array}$ \\
\hline \multicolumn{6}{|c|}{ Concentrators } \\
\hline $\begin{array}{l}1 \mathrm{LECS} \\
3 \mathrm{MECS}\end{array}$ & $\begin{array}{c}0.1-10 \\
1-10\end{array}$ & 0.5 & $1.5\left(\frac{E}{6}\right)^{-0.5}$ & $\begin{array}{c}20 \text { at } 0.25 \mathrm{keV} \\
150 \text { at } 6 \mathrm{keV}\end{array}$ & $8\left(\frac{E}{6}\right)^{-0.5}$ \\
\hline HPGSPC & $3-120$ & 1(coll.) & - & 450 (geom.) & $4\left(\frac{E}{60}\right)^{-0.5}$ \\
\hline PDS & $15-300$ & 1.5(coll.) & - & 800 (geom.) & $18\left(\frac{E}{60}\right)^{-0.5}$ \\
\hline $2 \mathrm{WFC}$ & $2-30$ & $20 \times 20\left({ }^{a}\right)$ & 5 & $250\left(^{a}\right)$ (through mask) & $18\left(\frac{E}{6}\right)^{-0.5}$ \\
\hline
\end{tabular}

(a) per unit.

\section{2. - The instruments}

The main characteristics of the SAX instruments (see also Butler and Scarsi, 1990 and references therein) are give in table I and fig. 2 .

\section{3. - The concentrator/spectrometer system}

The concentrator/spectrometer system consists of four separate concentrator mirror assemblies with a focal length of $185 \mathrm{~cm}$, each one with a position-sensitive, xenon-filled, GSPC in the focal plane, three covering the 1-10 keV range (MECS), the fourth one extending the range down to $0.1 \mathrm{keV}$ (LECS).

The mirror assemblies, for which the development and testing has been performed at IFCTR and IFCAI, are composed of 30 nested gold-coated mirrors, which are confocal double-cone approximations to a Woltjer 1 configuration. X-ray test on the prototype mirrors (Citterio et al., 1990, Conti et al., 1993; 1994) and on the flight unit in the Panter calibration facility have shown a resolution of $40^{\prime \prime}$ (HPR), better than the design goal of 1 arcmin and confirmed the prediction on the effective area (fig. 2). The concentrators are designed to maximize their effective area around $7 \mathrm{keV}$ for studies of iron line.

The four focal plane detectors are imaging Gas Scintillation Proportional Counters. Three of the GSPCs have $50 \mu \mathrm{m}$ beryllium windows, thus being sensitive in the range of 1-10 keV (ME) (Giarrusso et al., 1990, Bonura et al. 1992). The fourth GSPC developed by SSD/ESTEC (Parmar et al., 1990) is similar to the ME, except that a thin window (Bavdaz et al., 1994) allows for transmission of low-energy X-rays into the detector.

\section{4. - The high-energy instruments}

The high-energy instruments onboard SAX are, respectively, a 5 atm xenon-filled Gas Scintillation Proportional Counter (High-Pressure GSPC) and a NaI/CsI phoswich scintillator (Phoswich Detector System, PDS).

Great care has been put in the design to control any sources of systematic effects. Both instruments adopt the technique of rocking collimators, continuously pointing on and off source (with a period of the order of a minute) to monitor the background. Furthermore, in the case of PDS, which is composed of four units, the two halves of the experiment will 


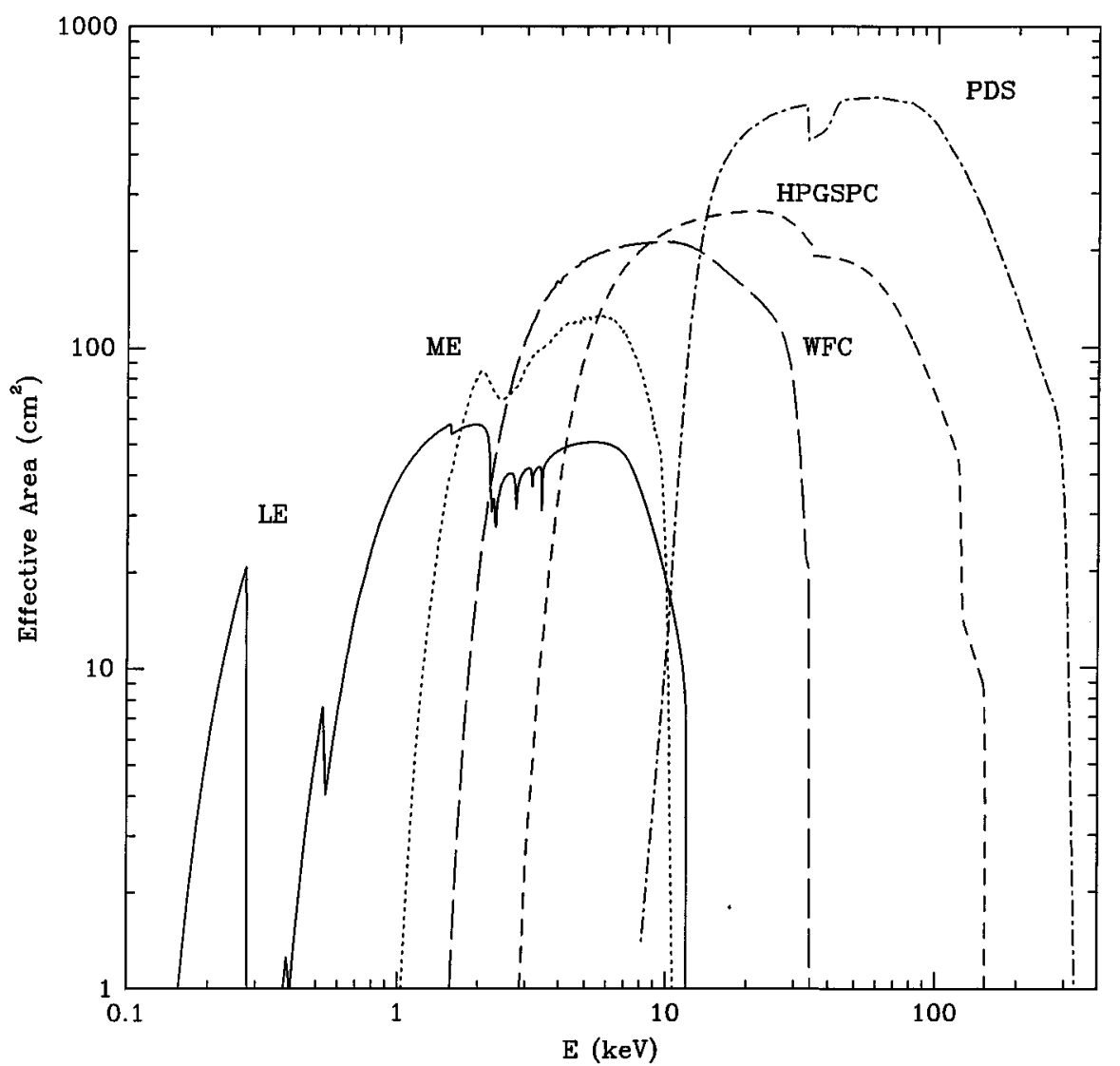

Fig. 2. - Effective area (scale on the right-hand side) of the SAX Narrow-Field Instruments. The continuous lines labelled as ME and LE give the effective, area of the three telescopes and of one telescope, respectively. The dotted lines give the focal plane detectors efficiency (left-hand scale).

alternatively point source and background regions, thus providing a continuous monitoring of the background. The two experiments have also an equalization gain system, based on tagged radioactive source (time-tagged for the PDS, position tagged for the HPGSPC), that will automatically adjust the high voltage in order to keep the gain of the detectors within $0.25-0.5 \%$.

The High-Pressure Gas Scintillation Proportional Counter is a new development of a GSPC filled with $5 \mathrm{~atm}$ Xenon/He mixture, which allows the detector to be sensitive up to $120 \mathrm{keV}$. The cell of the HPGSPC is seen by an Anger camera arrangement of seven photomultiplier tubes (PMT). The event position is used to correct the event energy with an overall improvement of a factor of two in energy resolution compared to proportional counters (Giarrusso et al., 1989).

The Phoswich Detector Sytem (PDS) consists of a square array of four independent $\mathrm{NaI}(\mathrm{Tl}) / \mathrm{CsI}(\mathrm{Na})$ phoswich scintillation detectors (Frontera et al., 1991). Each of the four detectors is made of the two crystals optically coupled and forming what is known as PHOSWICH (acronym of PHOSphor and sandWICH). The scintillation light produced in each phoswich is viewed, through a light guide of quartz, by a PMT. The NaI(Tl) acts as 


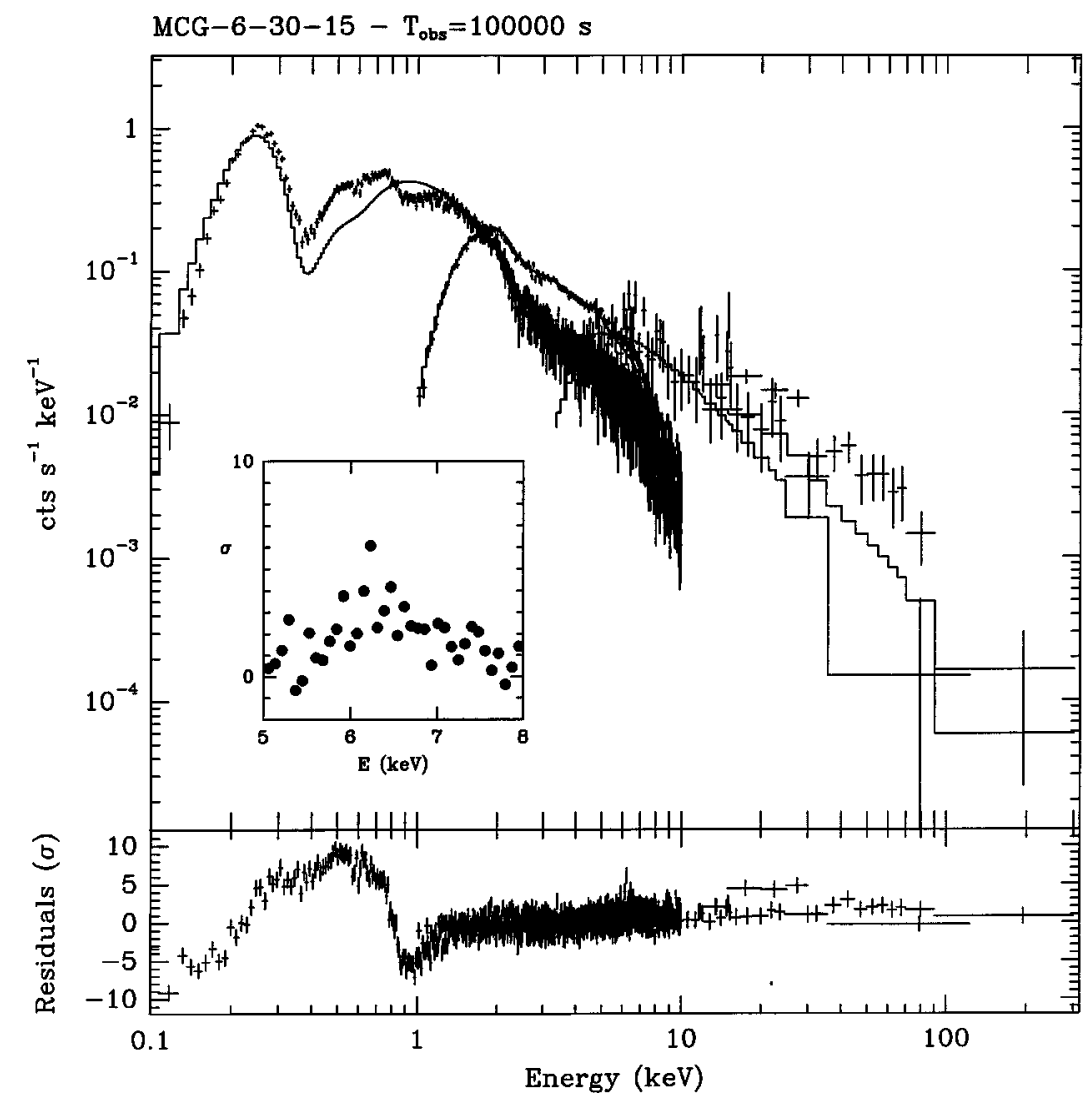

Fig. 3. - Spectrum from all the SAX-NFI in $T=10^{5} \mathrm{~s}$ for a Seyfert 1 like MCG-6-30-15. Note the sensitivity up to $200 \mathrm{keV}$.

$\mathrm{X}$-ray detector, while the $\mathrm{CsI}(\mathrm{Na})$ scintillator acts as an active shield. The phoswich configuration is well known to provide a detector with high efficiency at hard X-ray energies and a very low background level.

In order to further reduce the phoswhich background level, both a lateral and a top anticoincidence shield are provided. The lateral shielding system is made of $4 \mathrm{CsI}(\mathrm{Na})$ scintillators $10 \mathrm{~mm}$ thick that rejects a relevant fraction of the unwanted X-ray photons and charged particles impinging on the sides of the instruments. The top shield is made of an organic scintillator $1 \mathrm{~mm}$ thick that covers the X-ray entrance aperture. It rejects the background events due to charged particles, in particular high-energy electrons generated by interactions of cosmic rays with the materials of the instruments or with the satellite.

Two calibration systems are provided. One as gain control of each detection unit which is performed by using a time-tagged calibration source of ${ }^{241} \mathrm{Am}$. A continuous and automatic correction is performed by acting on the high-voltage supplies of the phoswich PMTs.

The other calibration system consists of two radioactive sources of ${ }^{57} \mathrm{Co}$ (lines at 14, $122,136 \mathrm{keV}$ ) distributed along a wire $141 \mathrm{~mm}$ long. Upon command, the sources, one for 


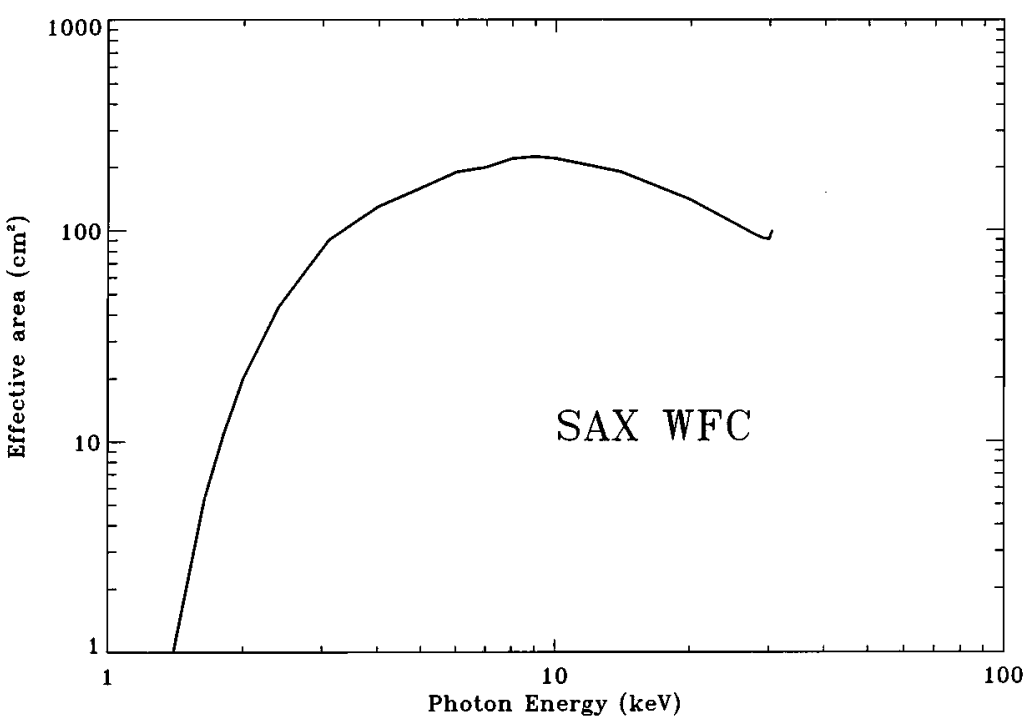

Fig. 4. - The effective area of a WFC.

each of the two detectors, cross the field of view of the instrument above the collimators and top shield. This monitoring allows to evaluate the absolute gain of the instrument and recalibrate it for long-term variations.

The lateral shields of the PDS are also used as detectors of gamma-ray bursts of cosmic origin (GRB) with fluence greater than $10^{-6} \mathrm{erg} \mathrm{cm}^{-2}$. The signals from each of the slabs in a programmable energy band, nominally $60-600 \mathrm{keV}$, are sent to both a Pulse Height Analyzer and the GRBM logic unit, where the trigger condition is checked. A GRB event accumulation is triggererd when at least two of the four lateral shields meet the trigger condition.

Note that all the Narrow-Field instruments share wide overlapping energy regions. This will allow a good relative calibration, essential to avoid artifacts in the deconvolution of spectra over the full range of SAX.

The sensitivity curves of all instruments can be found in Perola (1990). In fig. 3 we show the capability of NFI to determine the spectral shape of faint sources over the full range of 0.1-200 keV, in this case a Seyfert 1 like MCG-6-30-15.

\section{5. - The wide-field cameras}

The two identical Wide Field Cameras developed by SRON/SRU (Jager et al., 1989) consist of two position-sensitive proportional counters filled with $2 \mathrm{~atm}$ of xenon, which view the sky through a coded mask perpendicularly to the axis of the NFI and 180 degrees away from each other. They combine a large field of view $\left(20^{\circ} \times 20^{\circ}\right.$ per unit) with an angular resolution of 5 arcmin (FWHM). The energy band ranges from 1.8 to $30 \mathrm{keV}$ with a resolution of $18 \%$ at $6 \mathrm{keV}$. The effective area of one camera is shown in fig. 4 .

The sensitivity depends upon the pointing direction in the sky, because each source in the FOV contributes to the overall background. Towards high-galactic latitude the Cosmic Diffuse X-Ray Background is the main contributor to the background. In this case the sensitivity is of the order of a few mCrab in $10^{4}$. 


\section{6. - Scientific objectives}

The primary characteristics of SAX among past and future missions is its very wide spectral coverage, with well-balanced performances between the low-medium (0.1-10 $\mathrm{keV})$ and medium-high (1-200 keV) energy bands.

Given the instrument capabilities over the wide energy range described in the previous sections, SAX can provide a significant contribution (and a unique contribution for science involving the exploitation of the wide band) in several areas of X-ray astronomy such as compact galactic sources, active galactic nuclei, clusters of galaxies, supernova remnants, normal galaxies, stars and gamma-ray bursts.

With its minimum lifetime of two years (extendible to four years), SAX will be able to perform more than 2000 pointings. While the NFI will be the prime instruments most of the time, the WFC will be periodically used to scan the galactic plane to monitor the temporal behaviour of sources above $1 \mathrm{mCrab}$ and to detect transient phenomena; thanks to their large field of view, the WFC will be operated to monitor selected objects when the NFI perform their sequence of pointed observations. We expect to detect about 10-20 bright X-ray transients per year during the WFC observations. The observing program will be held flexible in order to accommodate those targets of opportunity for follow-up observations with the NFI.

The SAX program is the result of the efforts of so many and enthusiastic people and it is impossible to thank them all here. Let us honour the memory of the project manager M. CASCIOLA, who gave so much to the mission. Part of this paper was based on the contributions to the SAX Observers Handbook by C. Butler, L. Chiappetti, G. Conti, E. Costa, D. Dal Fiume, F. Frontera, S. Giarrusso, R. Jager, C. MaCCarone, G. Manzo, G. Matt, T. Mineo, A. Parmar, G. C. Perola, L. Piro, S. Re, B. SACCO, L. SAlotTi and A. SANTANGElo. Finally I wish to acknowledge the contributions to the mission of J. BleEker, G. BoElla, B. TAYlor, L. Scarsi; F. FaVata and M. TRIfoglio for the Ground Segment activities; G. MANARINI and B. NEGRI of ASI.

\section{REFERENCES}

Bavdaz M. et al., Nucl. Instrum. Methods, 345 (1994) 549.

Bonura A. et al., Proc. SPIE, 1743 (1992) 510.

Butler C. and Scarsi L., Proc. SPIE, 1344 (1990) 464.

Citterio O. et al., Nuovo Cimento C, 13 (1990) 375.

Conti G. et al., Proc. SPIE, 2011 (1993) 118.

Conti G. et al., Proc. SPIE, 2279 (1994) 101.

Frontera F. et al., Adv Space Res., 11 (1991) 281.

Giarrusso S. et al., Proc. SPIE, 1344 (1990) 106.

Giarrusso S. et al., Proc. SPIE, 1159 (1989) 514.

JAGER R. et al., Proc. SPIE, 1159 (1989) 2.

PARMAR A., SMith A. and BAVDAZ M., in Observatories in Earth orbit and Beyond, edited by Y. Kondo (Kluwer) 1990, p. 456.

Perola G. C., Adv. Space Res., 10 (1990) 295. 